\title{
COMPOSIÇÃO CORPORAL DE FRANGOS DE CORTE FÊMEAS COM 21 DIAS ALIMENTADAS COM NÍVEIS DE QUIRERA DE ARROZ NA DIETA
}

\author{
SILVA, Suelen Nunes da ${ }^{1}$; \\ BRUM JÚNIOR, Berilo de Souza ${ }^{1}$; \\ VALENTE, Beatriz Simões ${ }^{1}$; \\ MANZKE, Naiana Enhardt ${ }^{1}$; \\ LOPES, Débora Cristina Nichelle ${ }^{1}$; \\ RUTZ, Fernando ${ }^{1}$; \\ XAVIER, Eduardo Gonçalves ${ }^{1}$.
}

\section{RESUMO}

\begin{abstract}
A produção e a exportação de frangos de corte são atividades de grande importância econômica no Brasil, nesse sentido, a busca por alimentos que reduzam o custo da dieta, sem interferir no tempo para atingir o peso de abate, é constante. O presente estudo teve como objetivo verificar a influência de níveis de inclusão de quirera de arroz na dieta de frangos de corte, fêmeas, sobre a composição corporal. Foram utilizadas 160 fêmeas de frangos de corte da linhagem Cobb com um dia de idade, distribuídas em um delineamento inteiramente casualizado e divididas em quatro tratamentos: 0, 20, 40 e 60\% de quirera de arroz, com 10 repetições. As dietas foram isocalóricas, isoprotéicas e isoaminoacídicas. Aos 21 dias foram selecionadas as aves mais pesadas de cada boxe para a avaliação da alometria, sendo eutanasiadas e evisceradas manualmente. Foram avaliadas as seguintes variáveis: comprimento corporal; pesos relativos de perna, peito, asa, coração, fígado, moela, jejuno, duodeno e íleo; comprimentos de peito, perna, aparelho digestório e intestino delgado; e profundidade de peito. As variáveis não foram afetadas significativamente pelos níveis de inclusão de quirera de arroz na dieta, exceto o peso relativo da moela que reduziu linearmente com o aumento da inclusão da quirera até o nível de $60 \%$. Concluiu-se que a quirera de arroz pode ser incluída em até $60 \%$ na dieta sem afetar as características corporais de frangos de corte fêmeas abatidas com 21 dias de idade.
\end{abstract}

Palavras-chave: Alometria. Composição corporal. Moela. Peito. Peso relativo. 
O Brasil é o principal exportador mundial de carne de frango e o segundo maior produtor. No ano de 2018 foram produzidas 12,86 milhões de toneladas dessa proteína, sendo que o mercado interno consumiu $68,1 \%$ da produção e os restantes $31,9 \%$ foram para exportação (ABPA, 2019).

Uma maior disponibilidade e as oscilações nos preços dos grãos de cereais têm feito com que, cada vez mais, pesquisadores busquem fontes alternativas para a alimentação das aves (NUNES et al., 2011).

A quirera de arroz (QA), composta pelos grãos quebrados durante o beneficiamento do arroz (Oryza sativa L.), é um alimento com potencial para utilização na nutrição animal, substituindo o milho e reduzindo o custo da dieta. O Rio Grande do Sul é o maior produtor de arroz do Brasil, responsável por uma colheita de mais de 7,2 milhões de toneladas do grão na safra 2018/2019 (IRGA, 2019). Estima-se que 14\% desse total seja comercializado como quirera, podendo ser utilizado na composição de dietas para animais (SILVA; ACHERI, 2009).

A substituição do milho e do farelo de soja pode ser limitada pela composição bromatológica e, também, pela presença de fatores antinutricionais nos possíveis substitutos desses grãos (OLIVEIRA et al., 2000). O inibidor de tripsina, a hemaglutinina-lecitina e os fitatos são fatores antinutricionais encontrados no arroz que podem limitar seu uso, e de seus subprodutos, na nutrição animal (GUL et al., 2015; YOUNAS et al., 2011).

Dessa forma, o presente estudo objetivou avaliar a inclusão de diferentes níveis de quirera de arroz na dieta de frangos de corte, fêmeas, sobre as características corporais.

O experimento foi realizado no Aviário Experimental do Departamento de Zootecnia da Faculdade de Agronomia Eliseu Maciel da Universidade Federal de Pelotas. Foram utilizadas 160 fêmeas de frangos de corte da linhagem Cobb, com um dia de idade, distribuídas em um delineamento completamente casualizado com quatro tratamentos: 0, 20, 40 e 60\% de QA e 10 repetições de quatro aves cada uma. O período experimental foi de 21 dias.

As dietas experimentais (Tabela 1) foram formuladas para atender às necessidades da categoria, de acordo com a recomendação de Rostagno et al. (2005). As pintainhas foram 
alojadas em baterias metálicas com piso de grade, contendo comedouros metálicos tipo calha, bebedouros automáticos tipo nipple com copo e aquecimento com campânula a gás.

Tabela 1 - Composição centesimal e química de dietas pré-iniciais e iniciais para frangos de corte fêmeas.

PRÉ-INICIAL (1-7 dias)

INICIAL (8-21dias)

\begin{tabular}{lcccccccc}
\hline Ingredientes \% & QA 0 & QA 20 & QA 40 & QA 60 & QA 0 & QA 20 & QA 40 & QA 60 \\
Milho & 59,82 & 40,98 & 22,15 & 3,31 & 63,17 & 42,95 & 22,74 & 2,55 \\
Farelo de soja & 34,45 & 33,19 & 31,92 & 30,65 & 31,38 & 31,33 & 31,28 & 31,20 \\
Quirera de arroz & 0,00 & 20,00 & 40,00 & 60,00 & 0,00 & 20,00 & 40,00 & 60,00 \\
Óleo & 1,37 & 1,37 & 1,37 & 1,37 & 1,56 & 1,80 & 2,03 & 2,26 \\
Fosfato bicálcico & 1,95 & 1,99 & 2,03 & 2,07 & 1,81 & 1,84 & 1,88 & 1,91 \\
Calcário & 0,85 & 0,85 & 0,85 & 0,85 & 0,80 & 0,80 & 0,80 & 0,80 \\
Sal & 0,51 & 0,51 & 0,51 & 0,51 & 0,49 & 0,49 & 0,49 & 0,49 \\
Suplemento1 & 0,50 & 0,50 & 0,50 & 0,50 & 0,50 & 0,50 & 0,50 & 0,50 \\
DL-Metionina & 0,28 & 0,29 & 0,30 & 0,31 & 0,18 & 0,18 & 0,18 & 0,18 \\
L-Lisina & 0,22 & 0,24 & 0,27 & 0,29 & 0,11 & 0,10 & 0,10 & 0,09 \\
DL-Treonina & 0,04 & 0,08 & 0,11 & 0,14 & 0,00 & 0,00 & 0,00 & 0,00 \\
\hline & \multicolumn{7}{c}{ Composição analisada } \\
\hline EM Kcal/kg & 2950 & 2950 & 2950 & 2950 & 2890 & 2890 & 2890 & 2890 \\
Umidade \% & 11,60 & 10,91 & 11,90 & 11,95 & 12,32 & 12,23 & 12,38 & 11,9 \\
Extrato etéreo \% & 4,08 & 4,00 & 3,87 & 2,58 & 1,96 & 1,60 & 1,28 & 1,52 \\
Cinzas \% & 5,74 & 6,14 & 5,84 & 5,64 & 5,40 & 5,37 & 5,49 & 5,88 \\
Proteína bruta \% & 19,80 & 19,97 & 19,76 & 18,83 & 18,89 & 19,06 & 19,35 & 19,83 \\
Fibra bruta \% & 3,22 & 2,94 & 2,21 & 2,04 & 8,45 & 4,57 & 3,50 & 3,05 \\
\hline
\end{tabular}

QA-0: $0 \%$ de quirera de arroz; QA-20: $20 \%$ de quirera de arroz; QA-40: $40 \%$ de quirera de arroz; QA-60: $60 \%$ de quirera de arroz.

${ }^{1}$ Suplemento Vitamínico-Mineral: quantidade por kg de ração: Vit. A (UI): 12.000; Vit. D3 (UI): 4.500; Vit. E (mg): 24; Vit. K3 (mg): 3; Vit. B1 (mg): 3; Vit. B2 (mg): 8,5; Vit. B12 ( $\mu \mathrm{g})$ : 18; Ác. Fólico (mg): 1,5; Ác. Nicotínico (mg): 32; Ác. Pantotênico (mg): 24; Biotina ( $\mu \mathrm{g})$ : 250; $\mathrm{Mn}$ (ppm): 90; Zn (ppm): 80; Fe (ppm): 60; Cu (ppm): 12; I (ppb): 900; Se (ppb): 300; Salinomicina (ppm): 60; Bacitracina de zinco (ppm): 60; Olaquindox (mg): 50; Endox (ppm): 80.

${ }^{2}$ Análises realizadas no LNA/DZ/FAEM/UFPEL.

Aos 21 dias de idade foi selecionada, de cada unidade experimental, a ave com maior peso corporal. As aves escolhidas foram submetidas a anestesia inalatória com isoflurano e eutanasiadas por aprofundamento anestésico, para posterior realização da alometria. Após constatada a morte, as aves foram fixadas pelas patas em uma superfície plana com $60^{\circ}$ de inclinação e um peso de $400 \mathrm{~g}$ foi preso ao bico, distendendo-as para a obtenção do comprimento corporal. 
Manualmente, com o auxílio de uma tesoura, foi realizada a evisceração e a separação dos órgãos, do peito sem a pele, de uma perna (coxa + sobrecoxa sem pele) e das asas (com pele e sem as penas primárias e secundárias). As medidas, do comprimento do peito (realizada do início da bifurcação da quilha até o final da cartilagem esternal), da largura do peito e do comprimento de perna, foram obtidas com o auxílio de um paquímetro digital. $\mathrm{O}$ comprimento dos órgãos que compõe o aparelho digestório foi avaliado com fita métrica.

Os cortes e os órgãos foram pesados para a avaliação do peso relativo em função do peso corporal no momento do abate. Foram avaliados os seguintes pesos relativos: peito, perna, asa, coração, fígado, moela, duodeno, jejuno e íleo. Os dados foram submetidos à análise de variância e ao Teste de Tukey a $5 \%$ de significância, para comparação das médias e, posteriormente, à análise de regressão com o auxílio do programa estatístico Statistical Package for Social Sciences (SPSS).

Conforme pode ser observado na Tabela 2, os pesos relativos de perna, peito e asa, o comprimento corporal, o comprimento, a largura e a profundidade do peito e o comprimento da perna, não foram afetados pelos níveis de inclusão de quirera de arroz na dieta. Os pesos relativos de coração, fígado, duodeno, jejuno e íleo também não apresentaram diferença significativa entre os tratamentos (Tabela 3).

Tabela 2 - Pesos relativos de perna (PRPe), peito (PRPt) e asa (PRA), em g/kg; comprimento corporal (CC), comprimento (CPt), largura ( $\mathrm{LPt}$ ), profundidade de peito (PPt) e comprimento de perna (CPe), em milímetros, de frangos de corte fêmeas alimentadas com diferentes níveis de quirera de arroz e abatidas com 21 dias de idade.

\begin{tabular}{ccccccccc}
\hline QA (\%) & PRPe & PRPt & PRA & CC & CPt & LPt & PPt & CPe \\
\hline 0 & 8,57 & 19,41 & 6,97 & 421,1 & 110,23 & 67,40 & 17,26 & 108,57 \\
20 & 8,52 & 19,19 & 6,59 & 415,7 & 112,55 & 72,08 & 17,39 & 111,69 \\
40 & 8,57 & 19,37 & 6,69 & 414,4 & 109,50 & 69,34 & 16,23 & 109,12 \\
60 & 8,25 & 18,91 & 6,91 & 414,1 & 106,57 & 70,93 & 17,37 & 105,10 \\
\hline P & 0,26 & 0,91 & 0,81 & 0,70 & 0,72 & 0,14 & 0,61 & 0,12 \\
CV (\%) & 4,38 & 7,69 & 12,74 & 3,37 & 5,43 & 6,25 & 11,12 & 4,86 \\
\hline
\end{tabular}

QA: quirera de arroz; P: probabilidade; CV: coeficiente de variação.

* Médias seguidas de letras diferentes na mesma coluna diferem entre si pelo teste de Tukey. 
Tabela 3 - Pesos relativos de coração (PRC), fígado (PRF), duodeno (PRD), jejuno (PRJ) e íleo (PRI), em g/kg, de frangos de corte fêmeas alimentadas com diferentes níveis de quirera de arroz e abatidas com 21 dias de idade.

\begin{tabular}{cccccc}
\hline QA (\%) & PRC & PRF & PRD & PRJ & PRI \\
\hline 0 & 0,66 & 2,74 & 1,06 & 1,63 & 1,24 \\
20 & 0,66 & 3,18 & 0,92 & 1,60 & 1,18 \\
40 & 0,66 & 3,08 & 0,98 & 1,48 & 1,13 \\
60 & 0,63 & 3,47 & 1,05 & 1,75 & 1,13 \\
\hline P & 0,78 & 0,06 & 0,18 & 0,09 & 0,20 \\
CV (\%) & 9,22 & 16,31 & 13,21 & 12,86 & 10,64 \\
\hline
\end{tabular}

QA: quirera de arroz; P: probabilidade; CV: coeficiente de variação.

*Médias seguidas de letras diferentes na mesma coluna diferem entre si pelo teste de Tukey.

Brum Júnior et al. (2007) também não encontraram diferenças nos rendimentos de carcaça, fígado, coração, peito, coxa e sobrecoxa de frangos de corte aos 42 dias de idade, alimentados com dietas contendo até $40 \%$ de QA. Cancherini et al. (2008), ao avaliarem o desempenho e o rendimento de carcaças de frango de corte, aos 42 dias de idade, alimentados com dietas contendo subprodutos do arroz, concluíram que é possível usar o farelo de arroz integral e a quirera de arroz como ingredientes alternativos em dietas para frangos de corte, sem influenciar no consumo de ração, no ganho de peso e na conversão alimentar. No entanto, Men et al. (1996), em pesquisa com patos, utilizaram dietas com nível fixo de QA (ad libitum, 60 e $80 \mathrm{~g} / \mathrm{dia}$ ) e observaram que as aves alimentadas com maiores níveis de quirera na dieta apresentaram menores valores de rendimento de carcaça.

Wang et al. (2014), comparando dietas com arroz e milho para gansos, não encontraram diferença significativa entre elas nas variáveis: rendimento de carcaça eviscerada, rendimento de peito e de coxa e nem na gordura abdominal. Por outro lado, os mesmos autores encontraram maior peso relativo de jejuno e íleo, bem como intestinos delgado e grosso mais compridos nos animais que foram alimentados com dietas que continham arroz descascado, sugerindo ser o arroz um alimento que traz benefícios ao desempenho de aves de produção.

Amerah e Ravindran (2008) concluíram que o tipo de grão presente na dieta gera efeitos sobre a modificação do trato digestório de aves. Da mesma forma, Jorgensen et al. (1996) 
também relataram que os efeitos da fibra sobre a digestibilidade e o desenvolvimento do trato digestivo em aves dependem da fonte dos alimentos.

Os dados de todas as variáveis foram submetidos à análise de regressão. Apenas o peso relativo da moela apresentou um coeficiente de determinação alto $\left(R^{2}=0,975\right)$, a ponto de ter seu comportamento mais bem explicado pela regressão linear, conforme a Figura 1.

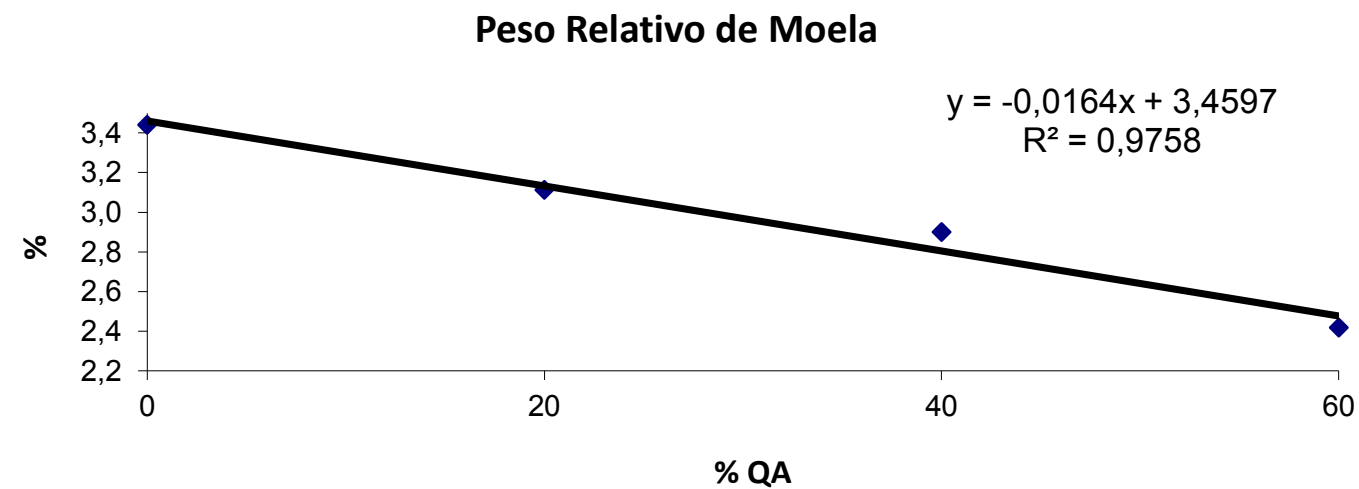

Figura 1 - Peso relativo de moela $(\mathrm{g} / \mathrm{kg}$ ) aos 21 dias de idade de frangos de corte fêmeas alimentadas com dietas contendo diferentes níveis de quirera de arroz (QA).

O rendimento de moela diminuiu linearmente com o aumento do nível de QA na dieta $(y=-$ 0,0164x+3,4597). Esses resultados sugerem que a QA apresenta maior degradabilidade e, com isso, ocorre menor desenvolvimento da musculatura da moela.

Hetland et al. $(2003,2004)$ relataram que o aumento de fibra na dieta de frangos e de poedeiras, favoreceu o desenvolvimento da moela. Isso pode explicar o comportamento linear do peso relativo desse órgão, no presente trabalho, o qual diminuiu conforme o teor de fibra na dieta foi sendo reduzido. Outros fatores que parecem tornar a quirera de arroz um alimento mais bem aproveitado pelos frangos e, dessa forma, diminuir o tamanho da moela, são a menor quantidade de amilose e de polissacarídeos não amiláceos encontrados no grão de arroz, em comparação com o de milho (NANTO et al., 2012). 
Diante do que foi apresentado, conclui-se que a quirera de arroz pode ser incluída em até $60 \%$ na dieta, sem afetar as características corporais de frangos de corte fêmeas abatidas com 21 dias de idade.

\section{BODY COMPOSITION OF 21-DAYS FEMALE BROILERS FED WITH LEVELS OF BROKEN RICE IN THE DIET}

\section{ABSTRACT}

$\mathrm{T}$ he production and export of broilers are activities of great economic importance in Brazil, in this sense, the search for foods that reduce the cost of the diet, without interfering in the time to reach the slaughter weight, is constant. The objective of this study was to verify the influence of inclusion levels of broken rice in the diet of female broilers on the body composition. A total of 160 one day old Cobb female broilers were used, distributed in a completely randomized design and divided in four treatments: 0, 20, 40 and $60 \%$ of broken rice, with 10 replicates. The diets were isocaloric, isoproteic and isoaminoacidic. At 21 days, the heaviest birds of each box were selected for the evaluation of allometry, being euthanized and eviscerated manually. The following variables were evaluated: body length; relative weight of leg, chest, wing, heart, liver, gizzard, jejunum, duodenum and ileum; length of chest, leg, digestive tract and small intestine; and chest depth. The variables were not significantly affected by broken rice inclusion levels in the diet, except for the relative weight of the gizzard that reduced linearly with the increase in the inclusion of broken rice up to the level of $60 \%$. It was concluded that broken rice can be included in up to $60 \%$ in the diet without affecting the body characteristics of broiler chickens slaughtered at 21 days of age.

Keywords: Allometry. Body composition. Chest. Gizzard. Relative weight. 


\section{COMPOSICIÓN CORPORAL DE POLLAS DE CARNE CON 21 DÍAS ALIMENTADAS CON NIVELES DE ARROZ QUEBRADO EN LA DIETA}

\section{RESUMEN}

a producción y exportación de pollos de engorde son actividades de gran importancia económica en Brasil, en este sentido, la búsqueda de alimentos que reduzcan el costo de la dieta, sin interferir en el tiempo para alcanzar el peso del sacrificio, es constante. El objetivo de este estudio fue verificar la influencia de los niveles de inclusión de arroz quebrado en la dieta de pollos de engorde sobre la composición corporal. Se utilizaron un total de 160 pollos de engorde de Cobb de un día de vida, distribuidos en un diseño completamente al azar y divididos en cuatro tratamientos: 0, 20, 40 y $60 \%$ de arroz quebrado, con 10 repeticiones. Las dietas fueron isocalóricas, isoproteínas e isoaminoácidas. A los 21 días, se seleccionaron las aves más pesadas de cada caja para la evaluación de la alometría, se las sacrificó y evisceró manualmente. Se evaluaron las siguientes variables: longitud corporal; peso relativo de la pierna, pecho, ala, corazón, hígado, molleja, yeyuno, duodeno y íleon; longitud del pecho, pierna, tracto digestivo e intestino delgado; y la profundidad del pecho. Las variables no fueron significativamente afectadas por los niveles de inclusión de arroz quebrado en la dieta, pero el peso relativo de la molleja disminuyó linealmente con el aumento de la inclusión de arroz al nivel de $60 \%$. Se concluyó que el arroz quebrado se puede incluir hasta en un $60 \%$ en la dieta sin afectar las características corporales de los pollos de engorde sacrificados a los 21 días de edad.

Palabras clave: Alometría. Composición corporal. Molleja. Pecho. Peso relativo.

\section{REFERÊNCIAS}

ABPA-ASSOCIAÇÃO BRASILEIRA DE PROTEÍNA ANIMAL. Relatório anual de 2019. Disponível em: <http://abpa-br.org/relatorios/> .

AMERAH, A. M.; RAVINDRAN, V. Influence of method of whole-wheat feeding on the performance, digestive tract development and carcass traits of broiler chickens. Animal Feed Science and Technology, v. 147, n. 4, p. 326-339, 2008.

BRUM JÚNIOR, B. S.; ZANELLA, I.; TOLEDO, G. S. P.; et al. Dietas para frangos de corte contendo quirera de arroz. Ciência Rural, v. 37, n. 5, p. 1423-1429, 2007. 
CANCHERINI, L. C.; DUARTE, K. F.; JUNQUEIRA, O. M.; et al. Desempenho e rendimento de carcaça de frangos de corte alimentados com dietas contendo subprodutos do arroz formuladas com base nos conceitos de proteína bruta e ideal. Revista Brasileira de Zootecnia, v. 37, n. 4, p. 616-623, 2008.

GUL, K.; YOUSUF, B.; SINGH, A. K.; et al. Rice bran: Nutritional values and its emerging potential for development of functional food-A review. Bioactive Carbohydrates and Dietary Fibre, v. 6, n. 1, p. 24-30, 2015.

HETLAND, H.; SVIHUS, B.; KROGDAHL, A. Effects of oat hulls and wood shaving on digestion in broilers and layers fed diets based on whole or ground wheat. British Poultry Science, $v$. 44. n. 2, p. 275-282, 2003.

HETLAND, H.; CHOCT, M.; SVIHUS, B. Role of insoluble non-starch polysaccharides in poultry nutrition. World's Poultry Science Journal, v. 60, n. 4, p. 415-422, 2004.

IRGA - INSTITUTO RIO GRANDENSE DO ARROZ. Boletim de Resultados da Lavoura - Safra 2018/2019 - Arroz Irrigado e Soja em Rotação. Boletim Técnico IRGA, 2019.

Disponível em: <https://irga-admin.rs.gov.br/upload/arquivos/201909/05171808-relatorioda-safra-2018-19-31-agosto-2019.pdf> .

JORGENSEN, H.; ZHOA, X. Q.; KNUDSEN, K. E.; et al. The influence of dietary fiber source and level on the development of the gastrointestinal tract, digestibility and energy metabolism in broiler chickens. British Journal of Nutrition, v. 75, n. 3, p. 379-395, 1996.

MEN, B. X.; OGLE, B.; PRESTON, T. R. Use of restricted broken rice in duckweed based diets for fattening Common and Muscovy ducks. Livestock Research for Rural, v. 8, n. 3, p. 20-25, 1996.

NANTO, F.; KIKUSATO, M.; ITO, C.; et al. Effect of dehulled, crushed and untreated wholegrain paddy rice on growth performance in broiler chickens. Japan Poultry Science Association, v. 49, p. 291-299, 2012.

NUNES, J. K.; GONÇALVES, F. M.; DALLMANN, H. M.; et al. Desenvolvimento do sistema digestório de frangos de corte alimentados com farinha de batata doce. Archivos de Zootecnia, v. 60, n. 232, p. 1105-1114, 2011.

OLIVEIRA, P. B.; MURAKAMI, A. E.; GARCIA, E. R. M.; et al. Influência de fatores antinutricionais da Leucena (Leucaena leucocephala e Leucaena cunningan) e do feijão Guandu (Cajamus cajan) sobre o epitélio intestinal e desempenho de frangos de corte. Revista Brasileira de Zootecnia, v. 29, n. 6, p. 1759-1769, 2000.

ROSTAGNO, H. S.; ALBINO, L. F. T.; DONZELE, J. L.; et al. Composição de alimentos e exigências nutricionais de aves e suínos. 2. ed. Viçosa: UFV, 2005. 186p. 
SILVA, R. F.; ASCHERI, J. L. R. Extrusão de quirera de arroz para uso como ingrediente alimentar. Brazilian Journal of Food Technology, v. 12, n. 3, p. 190-199, 2009.

WANG, Z. Y.; YANG, H. M.; LU, J.; et al. Influence of whole hulled rice and rice husk feeding on the performance, carcass yield and digestive tract development of geese. Animal Feed Science and Technology, v. 194, p. 99-105, 2014.

YOUNAS, A.; BHATTI, M. S.; AHMED, A.; et al. Effect of rice bran supplementation on cookie baking quality. Pakistan Journal of Agricultural Sciences, v. 48, n. 2, p. 129-134, 2011.

Autor para correspondência: Eduardo Gonçalves Xavier. Universidade Federal de Pelotas, Campus Universitário, Capão do Leão-RS. egxavier@yahoo.com 